\title{
Marked Hypofibrinogenemia and Gastrointestinal Bleeding After Copperhead (Agkistrodon contortrix) Envenomation
}

\author{
Kathryn T. Kopec, DO; May Yen, MD; Matthew Bitner, MD, MEd; C. Scott Evans, DO; Charles J. Gerardo, MD, MHS \\ From the Department of Emergency Medicine, Carolinas Medical Center, Charlotte, NC (Dr Kopec); Department of Emergency Medicine, \\ University of North Carolina, Chapel Hill, NC (Dr Yen); Division of Emergency Medicine, Duke University Medical Center, Durham, NC \\ (Dr Gerardo); Department of Emergency Medicine, Greenville Health System, Greenville, SC (Dr Bitner); and the Division of Emergency \\ Medicine, Palo Alto VA Medical Center, Palo Alto, CA (Dr Evans).
}

\begin{abstract}
Compared with other crotaline envenomations, copperhead envenomations have historically been reported as having less severe hematologic venom effects and rarely hemorrhage. We report a case of clinically significant gastrointestinal bleeding after a copperhead (Agkistrodon contortrix) envenomation. A 52-year-old woman with a history of systemic lupus erythematosus was bitten on her right medial ankle after which hypofibrinogenemia and hematochezia developed. The symptoms resolved after repeated administration of Crotalidae polyvalent immune Fab (ovine) antivenom. She was discharged without further complications 2 days later. Although copperhead envenomations are classically considered less severe than other crotaline envenomations, this case demonstrates the potential of the venom to produce clinically significant hematologic effects.
\end{abstract}

Key words: gastrointestinal bleeding, hypofibrinogenemia, copperhead, Agkistrodon contortrix, envenomation

\section{Introduction}

Crotaline snake envenomation syndromes can present with a variety of findings from local tissue damage to systemic and hematologic derangements. When compared with other crotalines, copperhead envenomations have reportedly been less severe. Although measurable derangements in laboratory studies are common, clinically significant bleeding is rare. ${ }^{1}$ We present a case of a copperhead envenomation that presented with marked hypofibrinogenemia and hematochezia.

\section{CASE PRESENTATION}

A 52-year-old woman came to a local emergency department (ED) after being bitten on her right medial ankle by a copperhead snake. The snake was identified by a family member as a copperhead, the only venomous snake species endemic to the region where the bite occurred. $^{2-5}$ The snake species was further verified by written communication (June 2015) with the Partners in Amphibian and Reptile Conservation biologist of the North Carolina Wildlife Resources Commission.

Corresponding author: Kathryn T. Kopec, DO, 2225 Hawkins Street, Apartment 316, Charlotte, NC 28203 (e-mail: ktkopec@gmail.com).
On arrival, the patient was anxious and nauseated but had normal vital signs. Her medical history included systemic lupus erythematosus (SLE) but she was not taking any medications (no nonsteroidal anti-inflammatory, antiplatelet, anticoagulant, or corticosteroid drugs). Her right lower extremity was edematous, erythematous, and ecchymotic to the level of her ankle, where she had 2 fang marks over the medial malleolus. She subsequently had a nonbloody emesis and a large bloody bowel movement. Her heart rate increased to 118 beats/min. She was administered a $2 \mathrm{~L}$ bolus of normal saline, $4 \mathrm{mg}$ odansetron, tetanus toxoid, and 4 vials of Crotalidae polyvalent immune Fab (ovine) antivenom (CroFab; Savage Laboratories, Melville, NY). Despite this therapy, she remained tachycardic and had another bloody bowel movement. Treatment continued with 4 additional vials of antivenom, and she was transferred to a local tertiary care center ED by ambulance.

On arrival to the tertiary care center ED, the patient remained slightly tachycardic with a heart rate of 101 beats/min and was hypertensive with a blood pressure of 171/89 mm Hg. Her right lower extremity demonstrated edema, erythema, and ecchymosis to just below the knee. Her initial laboratory values were significant for 
the following: fibrinogen $54 \mathrm{mg} / \mathrm{dL}$, prothrombin time 16 seconds, international normalized ratio 1.4 , platelets $243 \times 10^{9}$, hemoglobin $11.8 \mathrm{~g} / \mathrm{dL}$, white blood cell count $20.7 \times 10^{9}$, and lactate $2.8 \mathrm{mmol} / \mathrm{L}$. Soon thereafter she had another large, loose, bloody bowel movement. Owing to her continued hematochezia, she was administered an additional 6 vials of antivenom.

The patient was admitted to the intensive care unit, where she had an uneventful course without recurrent hemorrhage, hemodynamic instability, or need for additional antivenom. She was transferred to the medical floor on hospital day 2 and had no recurrence of bleeding or worsening of her hematologic abnormalities. Her laboratory values showed improvement and eventual normalization of her fibrinogen, international normalized ratio, prothrombin time, and d-dimer. Her hemoglobin and platelets levels remained stable. She did not have a colonoscopy, and the anatomic location of her lower gastrointestinal bleed was not localized. She was discharged home after 2 days without delayed bleeding, tissue necrosis, or other complications.

\section{Discussion}

Copperhead snakes (Agkistrodon contortrix) are members of the Crotalinae subfamily. Although measurable hematologic abnormalities are well documented in patients with crotaline envenomation, clinically significant hemorrhage is uncommon. ${ }^{6}$ Copperhead venom is less toxic, with a higher $\mathrm{LD}_{50}$ (the dose required to kill $50 \%$ of the population), as compared with other crotaline snake venom. ${ }^{7}$ Consequently, the clinical envenomation syndromes of the copperhead tend to be less severe, and bleeding is rare. ${ }^{1,8,9}$ Reports of several cohorts of patients with copperhead envenomation without any significant bleeding have been published. ${ }^{1}$ Only 1 report has been published, of a cohort of 178 patients presenting to a regional poison center, that included 2 patients with bleeding: a 2-year-old child who sustained multiple bites and became anemic secondary to hemorrhage into her envenomated limb; and a 36-year-old man with gastrointestinal bleeding. ${ }^{8}$ The rarity of clinically significant bleeding has prompted recommendations

for limitations both on laboratory testing and on treatment. ${ }^{1,10}$ However, useful predictors of clinically important hematologic abnormalities remain unclear.

In the case presented here, the severity of local tissue injury and systemic toxicity did not clearly predict the severity of the hematologic venom effects. The patient's local tissue symptom progression stopped below the knee; this degree of local tissue findings is common with copperhead envenomation and is not known to correlate with clinically significant bleeding. This patient also had systemic signs and symptoms (tachycardia and vomiting); although serious systemic symptoms are rare, these mild systemic findings are not uncommon with copperhead envenomation and are unlikely to be predictive of an increased risk of bleeding. In 1996, Dart et al. ${ }^{11}$ noted that none of the venom effect domains (local wound, cardiovascular, pulmonary, gastrointestinal, central nervous system, and hematologic) evaluated in the validation of the Snakebite Severity Score correlated highly with each other. In crotaline envenomation, a complex mixture of proteolytic enzymes, low molecular weight polypeptides, glycoproteins, and other distinct venom components cause the clinical venom effects. These molecules may exert individual toxic effects, work in combination, or produce numerous effects from a single toxin. ${ }^{12}$ Therefore, a patient may have a severe venom effect in 1 domain and not in another, depending on which venom components and toxic effects predominate.

The cause of hemorrhage in the patient described here is likely a combination of venom-, snake-, and patientspecific factors. As crotaline venoms contain toxins that both promote and inhibit platelet aggregation and are both procoagulant and anticoagulant, the balance of these effects determines which patients bleed. ${ }^{13}$ Snake subspecies may also play a role. It has been proposed that southern copperhead snakes may have greater venom effects than northern or broad-banded copperheads. ${ }^{14}$ Our patient had a history of SLE, which is typically associated with hypercoagulability, not hemorrhage. However, hypofibrinogenemia due to antifibrinogen antibodies or increased fibrinogen consumption associated with antiDNA positivity in SLE has been described. ${ }^{15}$ It is possible that this patient had a previously subclinical SLE-related coagulation disorder that was unmasked by the envenomation. Unfortunately, no additional hematologic or rheumatologic evaluation occurred during her hospitalization.

Our patient required a large amount of antivenom to obtain initial control of her envenomation syndrome. In a retrospective cohort study in $2011,{ }^{16}$ spontaneous hemorrhage was associated with difficulty achieving initial control. After controlling for other important variables, however, only thrombocytopenia and neurologic effects remained significantly associated in the study. ${ }^{16}$ As our patient had neither thrombocytopenia nor significant neurologic manifestations, it is likely that the small number of patients in that study and the colinearity between significant bleeding and thrombocytopenia masked a real and important association between significant spontaneous bleeding and difficulty achieving initial control. 


\section{Conclusion}

Although copperhead envenomations are typically considered less severe than other crotaline envenomations, the case described provides an example of an uncommon but important bleeding event after envenomation. This case adds to the existing evidence demonstrating that copperhead envenomation has the potential for significant clinical manifestations and should not be considered universally benign.

\section{References}

1. Ali AJ, Horwitz DA, Mullins ME. Lack of coagulopathy after copperhead snakebites. Ann Emerg Med. 2015;65:404-409.

2. Braswell AL, Palmer WM, Beane JC. Venomous Snakes of North Carolina. Raleigh, NC: North Carolina State Museum of Natural Sciences, NC Department of Environment and Natural Resources; 2003.

3. Dorcas ME. A Guide to the Snakes of North Carolina Davidson, NC: Davidson College; 2011.

4. Palmer WM, Braswell AL. Reptiles of North Carolina Chapel Hill, NC: UNC Press; 1995.

5. North Carolinas State Park Website. Available at: http:// www.ncparks.gov/kerr-lake-state-recreation-area/ecology. Accessed July 29, 2014.

6. Lavonas EJ, Kokko J, Schaeffer TH, Mlynarchek SL, Bogdan GM, Dart RC. Short-term outcomes after Fab antivenom therapy for severe crotaline snakebite. Ann Emerg Med. 2011;57:128-137.
7. Sanchez EE, Galan JA, Perez JC, Rodriquez-Acosta A, Chase PB, Perez JC. The efficacy of two antivenoms against the venom of North American snakes. Toxicon. 2003:41:357-365.

8. Thorson A, Lavonas EJ, Rouse AM, Kerns WP. Copperhead envenomations in the Carolinas. J Toxicol Clin Toxicol. 2003;41:29-35.

9. Ruha MA, Curry SC, Albrecht C, Riley B, Pizon A. Late hematologic toxicity following treatment of rattlesnake envenomation with Crotalidae polyvalent immune Fab antivenom. Toxicon. 2011;57:53-59.

10. Walker JP, Morrison RL. Current management of copperhead snakebite. J Am Coll Surg. 2011;212:470-474.

11. Dart RS, Hurlbut KM, Garcia R, Boren J. Validation of a severity score for the assessment of crotalid snakebite. Ann Emerg Med. 1996;27:321-326.

12. Angulo Y, Lomonte B. Biochemistry and toxicology of toxins purified from the venom of the snake Bothrops asper. Toxicon. 2009;54:949-957.

13. McCleary RJ, Kini RM. Snake bites and hemostasis/ thrombosis. Thromb Res. 2013;132:642-646.

14. Keyler DE, Vandevoort JT. Copperhead envenomations: clinical profiles of three different subspecies. Vet Hum Toxicol. 1999;41:149-152.

15. Sule Yasar Bilge N, Korkmaz C. Hypofibrinogenemia in a patient with systemic lupus erythematosus: always a bad sign? Or not. Lupus. 2011;20:660-662.

16. Yin S, Kokko J, Lavonas E, Mlynarchek S, Bogdan G, Schaeffer T. Factors associated with difficulty achieving initial control with Crotalidae polyvalent immune Fab antivenom in snakebite patients. Acad Emerg Med. 2011;18:46-52. 